\title{
MORTALIDADE POR NEOPLASIA MALIGNA MAMÁRIA NO RIO GRANDE DO SUL ENTRE OS ANOS DE 1995 E 2005
}

Vilma Constancia Fioravante dos Santos ${ }^{1}$, Maiana Pinheiro dos Santos ${ }^{1}$, Adriana Roese ${ }^{2}$

RESUMO: O câncer de mama é um dos maiores problemas de Saúde Pública do mundo. Este estudo, de natureza epidemiológica e transversal, analisou a mortalidade por neoplasia maligna mamária de mulheres entre 1995 e 2005 no Estado do Rio Grande do Sul, a partir dos dados do DATASUS/MS. Foram objetivos identificar a faixa etária com a maior taxa de mortalidade e com maior crescimento em número de óbitos por esta causa, bem como discutir ações desenvolvidas na promoção da saúde às mulheres, em relação à neoplasia mamária feminina. Os resultados apontam que a faixa etária de 70 a 79 anos possui a maior porcentagem de crescimento numérico de óbitos e as de 50 a 59 e 60 a 69 anos são as de maior índice de óbitos. A partir dos achados, discute-se a importância de Políticas Públicas eficazes na promoção da saúde e estratégias que diminuam as barreiras entre o sistema de saúde e população feminina.

PALAVRAS-CHAVE: Neoplasias da mama; Mortalidade; Promoção da saúde.

\section{MORTALITY FROM BREAST MALIGNANT NEOPLASMS IN RIO GRANDE DO SUL BETWEEN THE YEARS 1995 AND 2005}

\begin{abstract}
Breast cancer is a major public health problem in the world. This epidemiological and cross-examined study analyzed the mortality from breast malignancy in women between 1995 and 2005 in Rio Grande do Sul, from the DATASUS/MS available data. Other objectives were to identify the age group with the highest mortality rate and greater growth in number of deaths from this cause, and discuss actions taken in promoting health for women, in relation to breast cancer in women. The results indicate that the age group of 70-79 years has the highest percentage of growth in numbers of deaths and that at ages of 50-59 and 60-69 years there are the highest rates of deaths. From the findings, the importance of an effective public policy on health promotion and strategies that reduce the barriers between the health system and the female population are discussed.
\end{abstract}

KEYWORDS: Breast cancer; Mortality; Health promotion.

\section{MORTALIDAD POR NEOPLASIA MALIGNA MAMARIA EN RIO GRANDE DO SUL ENTRE LOS AÑOS DE 1995 Y 2005}

RESUMEN: El cáncer de mama es uno de los mayores problemas de Salud Pública del mundo. Este estudio, de naturaleza epidemiológica y transversal, ha analizado la mortalidad por neoplasia maligna mamaria de mujeres entre 1995 y 2005 en estado de Rio Grande do Sul, con base en los datos del DATASUS/MS. Los objetivos fueron identificar la franja etaria con mayor índice de mortalidad y con mayor crecimiento en número de óbitos por esta razón, así como discutir acciones desarrolladas en la promoción de la salud a las mujeres, acerca de la neoplasia mamaria femenina. Los resultados apuntan que la franja etaria de 70 a 79 años posee el mayor porcentaje de crecimiento numérico de óbitos y las de 50 a 59 y 60 a 69 años son las de mayor índice de óbitos. Por medio de eses datos, se discute la importancia de Políticas Públicas eficaces en la promoción de la salud y estrategias que disminuyan los obstáculos entre el sistema de salud y la población femenina.

PALABRAS CLAVE: Neoplasias de la mama; Mortalidad; Promoción de la salud.

\footnotetext{
${ }^{1}$ Acadêmica do Curso de Graduação em Enfermagem da Universidade Federal do Pampa-UNIPAMPA.

${ }^{2}$ Enfermeira. Doutoranda em Enfermagem na Escola de Enfermagem da Universidade Federal do Rio Grande do Sul-EE-UFRGS. Professora Assistente da área de Saúde Coletiva do Curso de Enfermagem da UNIPAMPA.
}

Autor correspondente:

Adriana Roese

Universidade Ferderal do Rio Grande do Sul

Rua Gomes Carneiro, 1 - 96010-610 - Pelotas-RS, Brasil

Recebido: 01/09/09

E-mail: adriroese@gmail.com

Aprovado: 14/07/10 


\section{INTRODUÇÃO}

O câncer de mama é um dos tumores malignos mais frequentes na mulher e, apesar de ser considerado de bom prognóstico, o seu diagnóstico precoce assume papel de grande relevância no que se refere à cura ${ }^{(1)}$. Denota-se, desta forma, a importância de ações em promoção da saúde baseadas em políticas universalistas que tomem como foco a redução das diferenças no acesso aos serviços de saúde. Partindo-se da identificação mais ampla dos problemas de saúde e da problematização da compreensão dos contextos de vida, vale ressaltar que o atual conceito de promoção da saúde supera o modelo proposto por algumas conferências internacionais de promoção da saúde que seguiam o modelo comportamental. Atualmente, segue-se a direção da construção de uma nova cultura no setor saúde que incorpore a educação, o saneamento básico, o trabalho e a preservação ambiental ${ }^{(2)}$.

Esse tipo de câncer tem se apresentado como um dos maiores problemas de Saúde Pública do mundo. No Brasil, faz-se necessária uma reflexão a respeito da desinformação das mulheres em relação à doença, ao estágio desta, quando diagnosticada, e à importância dos procedimentos de detecção precoce, uma vez que o câncer de mama superou, nos últimos anos, em incidência, o câncer de colo do útero, antes o de maior incidência entre as neoplasias femininas ${ }^{(3)}$. Acredita-se que a informação auxilie a mulher na busca pelo atendimento de saúde e proporcione uma detecção precoce e, consequentemente, haja melhor prognóstico.

Além disso, segundo os dados do Instituto Nacional do Câncer (INCA), para o ano de 2009, 9.500 novos casos de câncer de mama foram estimados apenas na Região Sul, com uma parcela de 4.880 casos no Estado do Rio Grande do $\mathrm{Sul}^{(4)}$. Esses dados demonstram a necessidade de se considerar as proporções futuras de mortalidade por esse agravo, pois, no Brasil, o câncer de mama é a maior causa de óbitos por câncer na população feminina, observando-se a possível relação entre faixa etária e exposição a fatores de riscos para o desenvolvimento do câncer de mama. Dessa forma, por haver um avanço na estrutura etária nacional ${ }^{(5)}$, acredita-se na possibilidade de os índices de mortalidade por esse agravo seguirem as tendências de aumento das taxas de incidência da doença.

A observação clínica e os estudos epidemiológicos têm levado à caracterização dos fatores que aumentam, relativamente, o risco de desenvolvimento do câncer de mama, e a identificação destes poderá propiciar intervenções em saúde por meio da formulação de políticas públicas mais amplas para as mulheres. Existem muitas variáveis biológicas que influenciam uma mulher a ter ou não câncer de mama, entre elas, é possível destacar a história familiar de câncer de mama, a menarca precoce, a menopausa tardia, a primeira gestação tardia, a nuliparidade e a doença mamária benigna, além da exposição à radiação ionizante e os hábitos de vida ${ }^{(6)}$.

Nesse contexto, é importante ressaltar que o avanço da ciência e da tecnologia pode oferecer diagnósticos com maior fidedignidade e em estágios mais precoces da doença. Dessa forma, o impacto da melhoria dos recursos diagnósticos ainda não pode ser quantificado antes de ser avaliado o acesso da população aos serviços de saúde ${ }^{(6)}$. Sabe-se que o acesso a serviços de saúde a partir da implantação do Sistema Único de Saúde (SUS) tem evoluído de maneira positiva, em comparação ao de anos anteriores, principalmente, no que se refere aos princípios de universalidade, que permite a toda mulher acessar o serviço em quaisquer dos níveis de assistência, e o de integralidade, em que a legislação garante assistência em qualquer nível de complexidade ${ }^{(7)}$. Reconhece-se que o atendimento de média complexidade não é satisfatório no País, sendo o acesso a esse nível de assistência extremamente dificultado, porém, ressaltase que houve uma melhora a partir da implantação do SUS, em relação ao antigo Instituto Nacional de Assistência Médica da Previdência Social (Inamps).

Logo, destaca-se que promover a saúde é uma atividade desafiadora para a equipe multidisciplinar, uma vez que isto denota uma percepção ampliada do conceito de saúde, fugindo de conceitos idealistas e partindo de percepções mais operacionais, considerando uma nova cultura de saúde ${ }^{(2)}$. Um dos agentes potenciais na promoção da saúde e na prevenção de doenças é o enfermeiro, sendo este citado como um importante profissional na implementação de ações em educação voltadas para a diminuição da exposição a fatores de risco. Esse profissional, durante o desenvolvimento de ações em saúde, pode conhecer, interpretar e se aproximar da realidade dos usuários, trabalhando a partir das singularidades; e favorecendo também as chances de diagnóstico precoce ${ }^{(6)}$. Acredita-se que o enfermeiro com esse entendimento consiga articular o trabalho intersetorial e interdisciplinar, promovendo a saúde da população.

O sucesso na luta contra o câncer de mama envolve a construção de um programa preventivo, 
eficaz e abrangente. Para países com poucos recursos destinados à Saúde Pública, o acesso a métodos diagnósticos tem algumas barreiras ${ }^{(8)}$. Desse modo, acredita-se que exista a necessidade de uma abordagem em saúde que atenda às demandas das usuárias e seja de fácil acesso, considerando a necessidade de exames diagnósticos conforme as faixas etárias, as condições de risco para o desenvolvimento da doença, o exame clínico das mamas e a promoção em saúde. Pensa-se que deva haver uma conscientização dos gestores quanto à necessidade de aumentar o acesso dos profissionais às tecnologias duras, mas também às tecnologias leves, sendo salientado o vínculo, o acolhimento e a corresponsabilização entre esses e os usuários $^{(9)}$. $\mathrm{O}$ incremento das tecnologias leves deve promover uma detecção precoce da doença e, por conseguinte, um melhor prognóstico para a mulher.

A escolha por essa problemática de estudo deve-se ao fato de se ter identificado que o aumento de novos casos de neoplasia mamária acompanha o alarmante aumento da mortalidade feminina no País, seguindo a tendência mundial, onde, de um milhão de casos novos de câncer de mama, 502 mil levariam a óbito $^{(4)}$. Essa situação pode ter inúmeras origens, tais como as dificuldades no acesso aos serviços de saúde ou, até mesmo, algumas falhas na atenção básica e de média complexidade. Dessa forma, os objetivos deste trabalho foram identificar a faixa etária com a maior taxa de mortalidade por neoplasia maligna mamária feminina e, também, a faixa etária com maior crescimento em número de óbitos por esta causa, bem como discutir ações desenvolvidas na promoção da saúde às mulheres em relação à neoplasia mamária feminina.

Acredita-se que o presente estudo seja relevante, pois soma esforços na reflexão acerca desta doença que leva mulheres à morte e demonstra a importância da inserção do profissional enfermeiro em práticas de saúde voltadas para a promoção da saúde.

\section{METODOLOGIA}

Este estudo é de natureza epidemiológica e transversal. Acredita-se que estudos dessa natureza possam permitir a avaliação do comportamento das taxas de mortalidade por neoplasia maligna mamária ao longo do tempo, em uma determinada população.

A coleta de dados secundários referentes às taxas de mortalidade por esse tipo de neoplasia foi realizada junto à base de dados do Departamento de Informática do Sistema Único de Saúde (DATASUS), do Ministério da Saúde ${ }^{(10)}$, sendo retirados do Sistema de Informação sobre Mortalidade (SIM). Optou-se por estudar as faixas etárias de zero a $29 ; 30$ a 39; 40 a 49; 50 a 59; 60 a 69; 70 a 79; e 80 anos e mais, as quais foram assim categorizadas pelo próprio Sistema de Informação consultado. O estudo compreende o período de 1995 a 2005, para ser traçada uma tendência da mortalidade pela causa citada, no Estado do Rio Grande do Sul, destacando-se que o referido Sistema de Informação em Saúde oferece a limitação de ter registros até o ano de 2006. A coleta de dados foi realizada no dia 5 de agosto de 2009 e revisada em 13 de abril de 2010.

O tempo de uma década foi escolhido para permitir a análise da repercussão da doença e traçar uma tendência de mortalidade por neoplasia maligna mamária feminina no Estado. Para melhor análise do fenômeno estudado, optou-se por não especificar Regiões ou Municípios. A partir da investigação dos números absolutos de óbitos por faixa etária, utilizouse a análise de tendência, que consiste no ajuste da reta que melhor representa os valores absolutos de óbitos por câncer de mama para cada faixa etária, no período estudado. Assim, cada reta possui uma equação e esta permite calcular o crescimento considerando todos os pontos, ou seja, todos os valores, neste caso, o número absoluto de óbitos por neoplasia maligna mamária.

Os aspectos éticos foram respeitados no presente estudo, sendo observadas as normas do Conselho Nacional de Saúde e considerada a Resolução n. $196 / 96^{(11)}$ referente às fontes consultadas e aos dados de domínio público disponíveis no endereço eletrônico do Sistema de Informações sobre Mortalidade, no Departamento de Informática do Sistema Único de Saúde, do Ministério da Saúde (SIM/MS/DATASUS).

\section{RESULTADOS E DISCUSSÃO}

A partir dos dados secundários coletados no SIM e analisados, foi elaborado um gráfico para melhor visualização dos dados coletados. Por conseguinte, a figura 1 demonstra, em dados absolutos, que as faixas de 50 a 59 e 60 a 69 anos são as de maior índice de óbitos por neoplasia mamária, no período de 1995 a 2005. A análise dos dados permitiu a confirmação de uma tendência linear de crescimento de algumas faixas etárias, cujas porcentagens relativas ao crescimento relativo foram calculadas a partir da tendência.

Desta forma, verificou-se que algumas faixas 
etárias apresentam tendência linear de crescimento e que outras oscilam de maneira irregular ao longo do tempo, não demonstrando ritmo de crescimento nem de recuo; ou seja, a reta formada pelos dados é horizontal. Aquelas que apresentaram tendência linear de crescimento tiveram suas respectivas porcentagens de crescimento relativo calculadas a partir dessa tendência.

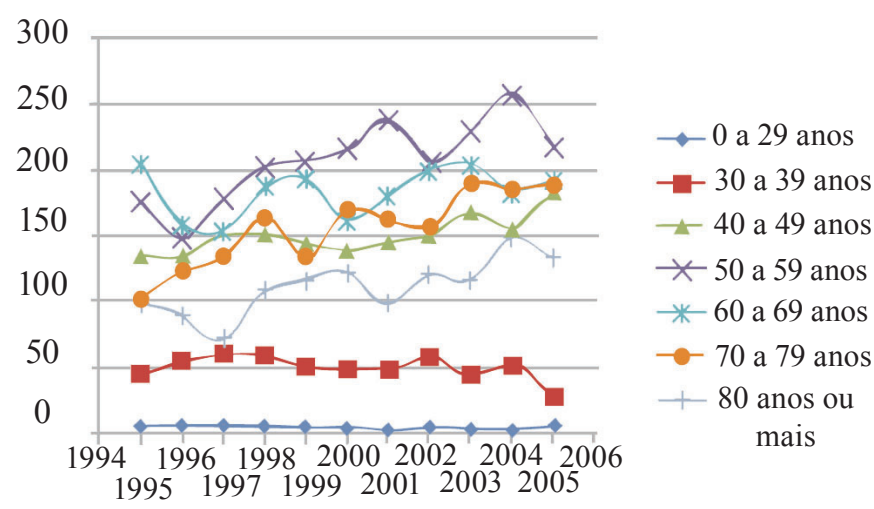

Fonte: Ministério da Saúde/SVS - Sistema de Informações sobre Mortalidade - SIM.

Figura 1 - Número de óbitos por neoplasia mamária feminina, por faixa etária, segundo o ano. Rio Grande do Sul, 1995-2005.

Para as faixas etárias de zero a 29 anos, 30 a 39 anos e 60 a 69 anos, não foi possível estabelecer uma tendência, em razão da grande variabilidade dos dados. Porém, é possível verificar que esses dados oscilam em torno de uma média corresponde de valores de óbitos, por ano, de 5; 52 e 183, respectivamente, de acordo com cada faixa etária. Destaca-se que, apesar de não ser possível o cálculo do crescimento linear na faixa etária de 60 a 69 anos, o número de mortes entre essas mulheres é alarmante. Para os demais dados, foi possível estabelecer uma tendência linear de crescimento e, por meio dela, é possível verificar um crescimento de $25,14 \%$ para a faixa etária de 40 a 49 anos; $45,26 \%$ para a faixa etária de 50 a 59 anos; $67,23 \%$ para a faixa etária de 70 a 79 anos e de $59,65 \%$ para a faixa a partir dos 80 anos de idade. Esses dados estão de acordo com a literatura consultada, a qual destaca que o câncer de mama é a maior causa de óbitos por câncer na população feminina, a partir dos 40 anos, o que comprova que a idade é um importante fator de risco ${ }^{(12)}$.

Apesar de as faixas etárias a partir dos 40 anos estarem em aumento progressivo, a faixa de 70 a 79 anos apresenta-se, majoritariamente, com o maior crescimento em número de óbitos por neoplasia mamária feminina entre as faixas etárias no período estudado, seguida da faixa a partir dos 80 anos e, depois, pelas faixas etárias de 50 a 59 anos e 40 a 49 anos, respectivamente. Nesse contexto, destaca-se o referencial teórico que retrata a ocorrência de casos esporádicos de câncer antes dos 35 anos de idade, e o aumento significativo observado nas faixas etárias mais elevadas, sendo descoberta, frequentemente, entre 40 e 60 anos $^{(6,12)}$.

A partir da análise das porcentagens relativas ao cálculo da tendência de crescimento, evidenciouse que as mulheres com mais de 50 anos foram as mais atingidas em relação à mortalidade por câncer de mama maligno no decorrer dos anos estudados, o que está em desacordo com o referencial bibliográfico estudado, o qual destaca que as mulheres, a partir dos 40 anos, são as mais atingidas ${ }^{(12)}$.

Questiona-se a razão do aumento da mortalidade por neoplasia mamária, visto que nesse período houve uma melhora na tecnologia de diagnóstico das neoplasias, uma maior qualificação profissional, bem como o desenvolvimento de programas em âmbito nacional voltados à saúde da mulher. Esses programas abordam várias condutas e material de apoio para atualização dos profissionais, tais como livros, folhetos explicativos e apostilas de atualização para o controle do câncer de mama ${ }^{(6)}$. Ressalta-se, também, a importância da publicação de estudos em periódicos científicos de circulação nacional e internacional, a fim de informar e instrumentalizar profissionais em suas ações na área da saúde. Assim, acredita-se que a tecnologia também possa ter aumentado o número de diagnósticos, o que talvez possa justificar o aumento no número de casos.

O Ministério da Saúde preconiza a realização do exame clínico anual das mamas, para todas as mulheres a partir de 40 anos de idade, e a realização de uma mamografia a cada dois anos, para mulheres de 50 a 69 anos. Além disso, o exame clínico é considerado como parte do atendimento integral à saúde da mulher, devendo ser realizado em todas as consultas clínicas, independente de faixa etária, podendo ser realizado por um profissional da saúde, médico ou enfermeiro ${ }^{(1)}$. Esse método tem se mostrado a estratégia mais eficaz na redução da mortalidade por câncer de mama ${ }^{(12)}$. A partir disso, questiona-se a abrangência das ações que envolvem a promoção da saúde e o acesso a esses métodos pelas mulheres, pois, como se verifica por meio da análise das porcentagens de crescimento e do gráfico exposto, o aumento, em número de mortes, por essa neoplasia, acomete majoritariamente as mulheres 
a partir dos 50 anos de idade. Em outras palavras, as evidências apontam que a identificação de mulheres que se encontram em estágio precoce do câncer de mama, diagnosticado por exame clínico das mamas, pode reduzir o número de óbitos por esse câncer ${ }^{(1,12)}$.

Destaca-se que a prevalência da realização do exame clínico das mamas está proporcionalmente ligada ao hábito da realização de consultas ginecológicas. Porém, discute-se o acesso a esses serviços, pois as mulheres que não se enquadram na faixa etária abonada pela indicação de rastreamento mamográfico foram aquelas que apresentaram maior tendência de crescimento em número de óbitos.

A relação entre o exame clínico das mamas e a realização de consultas ginecológicas pode ser ilustrada com os resultados de um estudo realizado com 879 mulheres na faixa etária dos 40 a 69 anos, o qual apontou que a prevalência de realização de consultas ginecológicas foi de $62 \%$ na amostra. Além disso, na faixa etária dos 40 a 49 anos, $70 \%$ das mulheres realizaram consulta ginecológica no último ano, $62,7 \%$ na faixa etária dos 50 a 59 anos e $44,2 \%$ na faixa etária dos 60 a 69 anos, sendo observado um decréscimo considerável no hábito de realizar exame clínico das mamas em decorrência do menor número de consultas ginecológicas realizadas com o avanço da idade ${ }^{(13)}$.

Além disso, quanto ao acesso das mulheres à prevenção secundária, ou seja, à detecção precoce do tumor quando o processo patológico já está instalado ${ }^{(14)}$, acredita-se que isso pode ser considerado como um fator determinante do quão avançada estará a doença quando descoberta, visto que, mesmo assintomáticas, as mulheres devem realizar consultas periódicas. $\mathrm{O}$ câncer de mama, muitas vezes, não causa dor, e a mulher apenas pode sentir um "caroço" que antes ela não percebia, podendo encontrar o câncer já num estágio bastante avançado. Pondera-se que a incidência de câncer de mama pode aumentar conforme as faixas etárias mais avançadas, observando-se a importância do desenvolvimento de ações educativas pelos profissionais que estão envolvidos, mobilizados e sensibilizados com ações de desmistificar o câncer e orientar a capacidade que a mulher tem de detectar essa doença ${ }^{(12)}$.

Em estudo realizado com 223 mulheres, observou-se que, do total de casos de câncer de mama diagnosticados, a forma de deteç̧ão por autoexame se deu em $76,7 \%$ da amostra, por exame clínico em $15,7 \%$ e por mamografia em 7,6\%. Esse resultado demonstra que a deteç̧ão de tumores ainda é realizada, prioritariamente, pela própria mulher e, em menor parcela, pelo exame clínico. Além disso, a detecção de tumores em fase inicial se deu em mais da metade da amostra por mamografia, e por autoexame e exame clínico, em 8,8 e $29,4 \%$, respectivamente ${ }^{(15)}$. Esses resultados estabelecem, de maneira clara, a importância da tríade autoexame, exame clínico e mamografia na luta contra o câncer de mama. Mesmo que o autoexame e o exame clínico das mamas tenham sido os métodos responsáveis pela detecção de 8,8 e 29,4\% dos tumores em fase inicial, a mamografia foi responsável pela maior parcela. Pode-se evidenciar a associação entre o método de detecção do tumor e o estadiamento do câncer de mama ${ }^{(15)}$.

Outro estudo identificou uma redução de $25 \%$ na mortalidade por câncer de mama com o rastreamento mamográfico de rotina ${ }^{(12)}$. Ressalta-se que a diminuição das barreiras, entre as mulheres, o rastreamento e a detecção precoces do câncer de mama podem resultar em ganhos para a mulher, para o Estado e para a sociedade $^{(15)}$. Consoante a isso, em estudo realizado em um município de São Paulo, 353 mulheres foram questionadas, via contato telefônico, sobre aspectos sociodemográficos, antecedentes pessoais e familiares de câncer de mama e oportunidades de diagnóstico precoce. Entre as mulheres entrevistadas, $81,1 \%$ responderam que estariam dispostas a realizar mamografia, caso esse exame fosse solicitado pelo profissional da saúde e estivesse disponível. Em contrapartida, a frequência de pedido de realização do exame é de $35,6 \%$ no referido município, representando as oportunidades de realização do exame ${ }^{(14)}$. Tal estudo retrata que o interesse das mulheres em realizar a mamografia pode ser contrariado pelo baixo acesso destas a esse tipo de serviço, ou seja, elas podem desejar realizar o exame diagnóstico, porém podem existir barreiras que as impedem de ter acesso a esse atendimento.

Esses dados trazem uma discussão acerca das possíveis disparidades existentes entre a consciência da importância da realização da mamografia e o acesso às mamografias. Destaca-se que esses dados são representados em baixas proporções, em comparação ao interesse das usuárias em realizá-los e sua relevância clínica em se tratando de precocidade diagnóstica.

À luz da discussão do acesso a serviços de saúde, é urgente a instituição de estratégias para corrigir atrasos entre intervalos compreendidos entre a mamografia e biópsia, resultado da biópsia e cirurgia e, por fim, resultado do exame anatomopatológico e tratamento adjuvante. Do ponto de vista oncológico, quanto 
maior o tempo transcorrido entre a cirurgia e o fim da quimioterapia neoadjuvante, mais comprometido fica o prognóstico oncológico da usuária. A detecção precoce da doença, nos serviços públicos, pode diminuir muito o tempo de encaminhamento para realização de exames de alta complexidade e tratamento adjuvante, reduzindo, assim, recursos financeiros que seriam gastos em tratamentos de câncer em estados mais avançados e, principalmente, a mortalidade em decorrência desse agravo $^{(16)}$. Mesmo porque, embora exista um grande desenvolvimento, em se tratando de cura, sua eficácia ainda depende da associação com o diagnóstico em fase precoce da doença. Dessa forma, reforça-se o papel da promoção da saúde nos espaços de Atenção Básica e a importância das ações dos profissionais que têm maior proximidade com as usuárias.

Acredita-se que esse crescimento na mortalidade por câncer de mama maligno feminino também possa estar relacionado ao adiamento da procura dessas mulheres pelos serviços de saúde. Ressaltase que as ações de promoção da saúde podem não estar abordando de maneira satisfatória as mulheres expostas aos maiores riscos e as questões que envolvam a necessidade da realização de exames clínicos e diagnósticos, destacando-se que, quanto mais tardia é a procura, mais reservado será o diagnóstico. Nesse sentido, a atuação do enfermeiro se torna desafiadora quando ele assume a função de reconhecer as particularidades dos usuários para poder traçar estratégias que busquem instigar, nas mulheres, o compromisso com a promoção de sua própria saúde ${ }^{(17)}$, bem como utilizar-se de estratégias que venham a abordar a mulher não apenas nos aspectos técnico e científico, mas também em seus aspectos psicoemocionais.

Os profissionais da saúde são atores importantes na promoção da saúde de todos os grupos sociais. O Ministério da Saúde, por meio da criação de programas como o Saúde da Família, atualmente denominado Estratégia da Saúde da Família (ESF), oferece uma assistência mais próxima da realidade dos usuários. Nesse espaço, os profissionais enfermeiros têm maior autonomia e possibilidade de fazer uso das diferentes abordagens em saúde, podendo reconhecer a realidade vivenciada pelas usuárias e planejar ações que contemplem suas demandas em saúde ${ }^{(6)}$. Esse fato pode aproximar a população, especialmente a feminina, do serviço de saúde, e com a promoção do vínculo, poder encaminhá-las precocemente aos serviços de apoio diagnóstico e de maior complexidade tecnológica.

\section{CONSIDERAÇÕES FINAIS}

A alta prevalência do câncer de mama faz com que estudos epidemiológicos de base populacional sejam de grande importância, pois essa doença ainda leva milhares de mulheres à morte, todos os anos. Enfatiza-se a necessidade de maior atenção a políticas públicas mais eficazes direcionadas para a promoção da saúde e para o diagnóstico precoce, especialmente voltadas àquelas mulheres que apresentam maiores riscos de desenvolver câncer de mama e com maiores índices de mortalidade por essa causa. Desse modo, seriam reduzidas as lacunas que existem entre elas e a detecção precoce.

Os registros disponibilizados na base de dados demonstraram que a faixa etária entre os 70 e 79 anos é a que possui a maior porcentagem de crescimento linear, seguida pela faixa etária de 80 ou mais anos e, depois, pela faixa etária de 50 a 59 anos. Além disso, com base nos valores absolutos apresentados no gráfico exposto, a faixa etária de 50 a 59 anos se fez a mais prevalente em número absoluto de óbitos por neoplasia maligna mamária, seguida pelas faixas etárias de 60 a 69 anos, 70 a 79 anos, 40 a 49 anos e pela de 80 ou mais anos. Quanto às faixas etárias anteriores, no período estudado, mantiveram-se estáveis a faixa etária de zero a 29 anos e a faixa de 30 a 39 anos, corroborando o referencial pertinente. Esses dados apontam a necessidade de um olhar mais crítico e abrangente acerca das questões de saúde que envolvem as mulheres em suas diferentes faixas etárias.

A principal meta frente ao câncer de mama é identificar seus fatores de risco para que seja possível trabalhar enfatizando-se a prevenção desses fatores. Por outro lado, se o processo patológico já estiver instalado, o objetivo será identificar o tumor o mais precocemente possível, para que o tratamento seja iniciado com brevidade, potencializando as chances de cura. A promoção da saúde e o diagnóstico precoce são as principais forças na luta contra o câncer, especialmente por meio de uma estratégia efetiva. $\mathrm{O}$ exame de mamas, por exemplo, é um exame simples, sem custos e que pode salvar milhões de vidas, sendo ele realizado, prioritariamente, por um profissional de saúde ou pela própria usuária.

O presente estudo trouxe uma reflexão a respeito de um grave problema de Saúde Pública que acomete mulheres de todas as partes do mundo, e esse cenário também faz parte da realidade do Estado do Rio Grande do Sul. O câncer de mama, por vezes, é uma doença silenciosa, cujo tratamento pode acabar 
sendo feito apenas quando a possibilidade de cura já é reduzida. Acredita-se, ainda, que a informação seja o melhor instrumento para promover ações que sensibilizem profissionais e usuárias para a detecção precoce da doença, diminuindo a barreira que existe entre a mulher e o sistema de saúde, reduzindo os altos índices de mortalidade por esta doença.

\section{REFERÊNCIAS}

1. Ministério da Saúde (BR). Controle do câncer de mama: documento de consenso. Rio de Janeiro: Instituto Nacional do Câncer, 2004.

2. Stotz EN, Araujo JWG. Promoção da saúde e cultura política: a reconstrução do consenso. Saúde Soc. 2004;13(2):5-19.

3. Gonçalves SMCM, Dias MR. A prática do auto-exame em mulheres de baixa renda: um estudo de crenças. Estud Psicol. 1999;4(1):141-59.

4. Ministério da Saúde (BR). Secretaria de Atenção à Saúde. Instituto Nacional do Câncer. Coordenação de Prevenção e Vigilância de Câncer. Estimativas 2008: Incidência de Câncer no Brasil. Rio de Janeiro; 2007.

5. Basílio DV, Mattos IE. Câncer em mulheres idosas das regiões sul e sudeste do Brasil: evolução da mortalidade no período de 1980-2005. Rev Bras Epidemiol. 2008; 11(2):204-14.

6. Ministério da Saúde (BR). Ações de enfermagem para o controle do câncer: uma proposta de integração ensino-serviço. 2a ed. Rio de Janeiro; Instituto Nacional do Câncer, 2002.

7. Conselho Estadual de Saúde (RS). SUS é legal - Rio Grande do Sul - Legislação federal e estadual. Porto Alegre; 2000.

8. Marinho LAB, Gurgel MSC, Cecatti JG, Osis MJD. Conhecimento, atitude e prática do auto-exame das mamas em centros de saúde. Rev Saúde Pública. 2003;37(5):576-82.

9. Merhy EE. Saúde: a cartografia do trabalho vivo. São Paulo: Hucitec; 2002.

10. Ministério da Saúde (BR). Departamento de Informática do Sistema Único de saúde. [acesso em 5 ago 2009]. Disponível: http://www.datasus.gov.br

11. Ministério Nacional da Saúde. Conselho Nacional de Saúde. Diretrizes e normas regulamentadoras de pesquisa envolvendo seres humanos. Resolução $n$. 196/96 de 10 de outubro de 1996. Brasília, 1996;4(2):1525.

12. Thuler, LC. Considerações sobre a prevenção do câncer de mama feminino. Rev Bras Cancerol. 2003;49(4):227-38.

13. Sclowitz ML, Menezes AMB, Gigante DP, Tessaro S. Condutas na prevenção secundária do câncer de mama e fatores associados. Rev Saúde Pública. 2005;39(3):340-9.

14. Molina L, Dalben L, Luca LA. Análise das oportunidades de diagnóstico precoce para as neoplasias malignas de mama. Rev Assoc Med Bras. 2003; 49(2):185-90.

15. Batiston AP, Tamaki EM, Santos MLM, Cazola LHO. Método de detecção do câncer de mama e suas implicações. Cogitare Enferm. 2009;14(1):59-64.

16. Trufelli DC, Miranda VC, Santos MBB, Fraile NMP, Pecoroni PG, Gonzaga SFR, et al. Análise do atraso no diagnóstico e tratamento do câncer de mama em um hospital público. Rev Assoc Med Bras. 2008;54(1):726.

17. Alcântara LFFL, Malveira EAP, Beque GV. Enfermeiras cuidando em oncologia ambulatorial: a consulta de enfermagem e o sentido do cuidar. Rev Enferm UERJ. 2004;12(3):259-64. 